\title{
NOTE ON THE STRUCTURE OF FIXED POINT SETS OF 1-SET-CONTRACTIONS ${ }^{1}$
}

\author{
W. V. PETRYSHYN
}

\begin{abstract}
Let $X$ be a real Banach space, $D$ a bounded open subset of $X$, and $T$ a demicompact 1-set-contraction of the closure $D$ into $X$. It is shown that under certain conditions the set $F(T)$ of fixed points of $T$ in $D$ is a continuum (i.e., $F(T)$ is a nonempty, compact and connected set).
\end{abstract}

The purpose of this short note is to extend the validity of Theorem 5 in [2] concerning the structure of fixed point sets to demicompact 1-setcontractions and thus to obtain a new result (see Theorem 1 below) which unifies and includes Theorems 4 and 5 in [2] as its special cases. For the detailed description of the problem, precise statements of the mentioned results, definitions, and the precise references to relevant contributions of various authors including those of Aronszain, Stampacchia, Krasnoselsky and Sobolevsky, Browder and Gupta, Vidossich, Deimling, and others, see [2].

Let $X$ be a real Banach space and $D$ a bounded open subset of $X$, with $\bar{D}$ and $\dot{D}$ denoting its closure and boundary, respectively. In what follows we shall need the following definitions and results (see [1], [2], [3]). We say that $\gamma(D)$ is the measure of noncompactness of $D$ if $\gamma(D)=$ $\inf \{d>0 \mid D$ can be covered by a finite number of sets of diameter less than or equal to $d\}$; a continuous mapping $T$ of $\bar{D}$ into $X$ is $k$-setcontractive if $\gamma(T(D)) \leqq k \gamma(D)$ for some $k \geqq 0$ and condensing if $\gamma(T(D))<\gamma(D) ; T: \bar{D} \rightarrow X$ is demicompact if each sequence $\left\{x_{n}\right\} \subset \bar{D}$ has a convergent subsequence $\left\{x_{n}\right\}$ whenever $\left\{x_{n}-T\left(x_{n}\right)\right\}$ is a convergent sequence in $X$. Clearly, $k$-set-contractions, $k<1$, and condensing mappings form a proper subclass of the class of demicompact 1-set contractions.

Using the degree theory for the translations of $k$-set-contractions with $k<1$ developed by Nussbaum [1], in Lemma 1 below we define and

Received by the editors October 15, 1970.

AMS 1970 subject classifications. Primary 47H10; Secondary 55C25.

Key words and phrases. Structure of fixed point sets, continuum, measure of noncompactness, 1-set-contractions, condensing, demicompact, generalized degree.

${ }^{1}$ Supported in part by the NSF Grant GP-20228 and in part by the Research Council of Rutgers University while the author was on the faculty research fellowship. 
establish certain properties of the degree for the translations of demicompact 1-set-contractions. We add that Lemma 1 can also be deduced (but not trivially) from the results of Nussbaum [1] on the fixed point index for "admissible" maps defined on certain metric absolute neighborhood retracts.

Let $D$ be a bounded open subset of $X, T$ a demicompact 1-set-contraction of $\bar{D}$ into $X$, and $f$ a given element in $X$ such that $(I-T)(x) \neq f$ for $x$ in $\dot{D}$. Since $T$ is demicompact, there exists $\delta>0$ such that $\|(I-T)(x)-f\| \geqq \delta$ for $x$ in $\dot{D}$. If $W: \bar{D} \rightarrow X$ is any $k$-set-contraction with $k<1$ such that $\|T(x)-W(x)\|<\delta$ for $x$ in $\dot{D}$, then $(I-W)(x) \neq f$ for $x$ in $\dot{D}$ so that, by the results in [1] for $k$-set-contractions with $k<1$, $\operatorname{deg}(I-W, D, f)$ is well defined and possesses most of the properties of the Leray-Schauder degree for compact displacements. We use this degree to define the degree of $I-T$ on $D$ over $f$ by the equality

$$
\operatorname{deg}(I-T, D, f)=\operatorname{deg}(I-W, D, f) .
$$

Since $T$ is 1-set-contraction, it follows that $W$ having the above properties always exist. Furthermore, $\operatorname{deg}(I-T, D, f)$ given by (i) is independent of the map $W$ which is chosen to approximate $T$. For $\operatorname{deg}(I-T, D, 0)$ the following holds.

LemMa 1. Let $D$ be an open bounded subset of $X$ and $T$ a demicompact 1-set-contraction of $\bar{D}$ into $X$ such that $T(x) \neq x$ for $x$ in $D$. Then $\operatorname{deg}(I-T, D, 0)$ is well defined and has the following properties:

(a) If $\operatorname{deg}(I-T, D, 0) \neq 0$, then $T$ has a fixed point in $D$.

(b) If $0 \notin(I-T)(\bar{D})$, then $\operatorname{deg}(I-T, D, 0)=0$.

(c) If $S=\{x \in D \mid(I-T)(x)=0\} \subset D_{1} \cup D_{2}$, where $D_{1}$ and $D_{2}$ are disjoint open subsets of $D$, then $\operatorname{deg}(I-T, D, 0)=\operatorname{deg}\left(I-T, D_{1}, 0\right)+$ $\operatorname{deg}\left(I-T, D_{2}, 0\right)$.

(d) If $F(x, t)=F_{t}: \bar{D} \times[0,1] \rightarrow X$ is a continuous mapping such that $\left\|x-F_{t}(x)\right\| \geqq \delta>0$ for $x$ in $\dot{D}$ and $t$ in $[0,1]$ and $\gamma(F(A \times[0,1])) \leqq$ $\gamma(A)$ for any $A \subset \bar{D}$, then $\operatorname{deg}\left(I-F_{t}, D, 0\right)$ is constant in $t \in[0,1]$.

Proof. (a) Let $W_{n}=k_{n} T$ be a $k_{n}$-set-contraction of $\bar{D}$ into $X$ with $k_{n}<1$ for each $n$ and $k_{n} \rightarrow 1$ as $n \rightarrow \infty$. Then

$$
d_{n}=\sup \left\{\left\|W_{n}(x)-T(x)\right\| \mid x \in \bar{D}\right\} \rightarrow 0
$$

as $n \rightarrow \infty$ and hence there exists an $N \geqq 1$ such that $d_{n}<\delta$ for $n \geqq N$. Thus (a) implies that $\operatorname{deg}\left(I-W_{n}, D, 0\right)=\operatorname{deg}(I-T, D, 0) \neq 0$ for $n \geqq N$. Hence, by the results in [1], $W_{n}$ has a fixed point $x_{n}$ in $D$ for each $n \geqq N$; therefore $\left\|x_{n}-T\left(x_{n}\right)\right\| \leqq d_{n} \rightarrow 0$ as $n \rightarrow \infty$. Since $T$ is demicompact and continuous, there exist a subsequence $\left\{x_{n_{j}}\right\}$ and an $x_{0}$ in $\bar{D}$ such that $x_{n_{5}} \rightarrow x_{0}$ and $T\left(x_{0}\right)=x_{0}$. Since $\operatorname{deg}(I-T, D, 0)$ is defined, $x_{0}$ lies in $D$. 
(b) This assertion follows from (a).

(c) Let $S_{i}=S \cap D_{i}$ and note that $S_{i}$ is closed for $i=1,2$, Let $U_{i}$ be an open neighborhood of $S_{i}$ such that $\bar{U}_{i} \subset D_{i}$ for $i=1,2$, If $\delta=\inf \left\{\|x-T(x)\| \mid x \in \bar{D}-U_{1} \cup U_{2}\right\}$, then $\delta>0$ and if we let $W$ be a $k$-set-contraction of $\bar{D}$ into $X$ with $k<1$ such that $\|T(x)-W(x)\|<\delta$ for $x$ in $\dot{D}$, then, by (i), $\operatorname{deg}(I-T, D, 0)=\operatorname{deg}(I-W, D, 0)$. Since $S_{W}=\{x \in D \mid W(x)=x\} \subset U_{1} \cup U_{2} \subset D_{1} \cup D_{2}$, the results in [1] imply that $\operatorname{deg}(I-W, D, 0)=\operatorname{deg}\left(I-W, D_{1}, 0\right)+\operatorname{deg}\left(I-W, D_{2}, 0\right)$ from which (c) follows because, as is easy to see, $\operatorname{deg}\left(I-W, D_{i}, 0\right)=$ $\operatorname{deg}\left(I-T, D_{i}, 0\right)$ for $i=1,2$,

(d) Let $F(x, t) \equiv F_{t}(x): \bar{D} \times[0,1] \rightarrow X$ satisfy the conditions of (d). Since $\left\|F_{t}(x)\right\| \leqq M$ for $(x, t) \in \bar{D} \times[0,1]$ and some $M>0$, it follows that if $k \in(1-(\delta / 2 M), 1)$ and $H_{t}: \bar{D} \times[0,1] \rightarrow X$ is given by $H_{t}(x)=$ $k F_{t}(x)$, then for all $x$ in $\bar{D}$ and $t \in[0,1]$ we have $\left\|H_{t}(x)-F_{t}(x)\right\| \leqq$ $(1-k) M<\delta / 2$ and for $x$ in $\dot{D}$ and $t \in[0,1]$ we have $\left\|x-H_{t}(x)\right\| \geqq$ $\left\|x-F_{t}(x)\right\|-\left\|F_{t}(x)-H_{t}(x)\right\| \geqq \delta / 2>0$; furthermore,

$$
\gamma(H(A \times[0,1]))=k \gamma(F(A \times[0,1])) \leqq k \gamma(A)
$$

for every $A \subset \bar{D}$. Hence, by the results in [1] for $k$-set-contractions with $k<1$ we have that $\operatorname{deg}\left(I-H_{t}, D, 0\right)$ is constant in $t \in[0,1]$ and so is $\operatorname{deg}\left(I-F_{t}, D, 0\right)$ since $\operatorname{deg}\left(I-F_{t}, D, 0\right)=\operatorname{deg}\left(I-H_{t}, D, 0\right)$ for $t \in[0,1]$. Q.E.D.

Now, using Lemma 1 and the arguments analogous to those in [2], we obtain the following generalization of Theorem 5 in [2] concerning the structure of the set $F(T)$ of fixed points of $T$ in $D$, with $T$ a demicompact 1-set-contraction.

THEOREM 1. Let D be a bounded open subset of a general Banach space $X$ and let $T$ be a demicompact 1-set-contraction of $\bar{D}$ into $X$ such that $\operatorname{deg}(I-T, D, 0) \neq 0$. Suppose there exists a sequence $\left\{T_{n}\right\}$ of condensing mappings of $\bar{D}$ into $X$ such that

(a) $\delta_{n}=\sup \left\{\left\|T_{n}(x)-T(x)\right\|, x \in \bar{D}\right\} \rightarrow 0$ as $n \rightarrow \infty$,

(b) the equation $x=T_{n}(x)+y$ has at most one solution in $D$ if $\|y\| \leqq \delta_{n}$.

Then the set $F(T)$ of fixed points of $T$ in $D$ is a continuum (i.e., $F(T)$ is nonempty, compact, and connected).

Proof. Since $\operatorname{deg}(I-T, D, 0) \neq 0$, Lemma 1 (a) implies that $F(T) \neq \varnothing$. Furthermore, $F(T) \subset D$ is a compact set since $T$ is demicompact on $\bar{D}$. Thus it remains to show that $F(T)$ is connected. Suppose then that $F \equiv F(T)$ is not connected. Then there exist nonempty disjoint compact sets $F_{1}$ and $F_{2}$ in $D$ such that $F=F_{1} \cup F_{2}$ and $d\left(F_{1}, F_{2}\right)=$ $s>0$. Let $D_{1}$ and $D_{2}$ be two disjoint open subsets of $D$ such that 
$D_{1} \supset F_{1}, D_{2} \supset F_{2}$, and $F \subset D_{1} \cup D_{2}$. It follows from Lemma 1(c) with $S=F$ that

$$
\operatorname{deg}(I-T, D, 0)=\operatorname{deg}\left(I-T, D_{1}, 0\right)+\operatorname{deg}\left(I-T, D_{2}, 0\right) .
$$

We shall derive our contradiction by showing that $\operatorname{deg}(I-T, D, 0)=$ $\operatorname{deg}\left(I-T, D_{2}, 0\right)=0$ while $\operatorname{deg}(I-T, D, 0) \neq 0$ by assumption.

Now, since $T$ is a demicompact mapping of $\bar{D}$ into $X, I-T$ is a closed mapping of $\bar{D}$ into $X$ which obviously has no zeros on $D^{\prime}=\bar{D}-$ $D_{1} \cup D_{2}$. Hence there exists a constant $\beta>0$ such that

$$
\|x-T(x)\| \geqq \beta \text { for all } x \text { in } D^{\prime} .
$$

Furthermore, since $T$ is a 1-set-contraction, $T(\bar{D})$ is bounded and $\|T(x)\| \leqq m$ for all $x$ in $\bar{D}$ and some $m>0$. If we take a fixed $k$ such that $1-(\beta / 2 m)<k<1$ and define $H(x)=k T(x)$ for all $x$ in $\bar{D}$, then we have $\operatorname{deg}(I-T, D, 0)=\operatorname{deg}(I-H, D, 0)$. Now, since

$$
\alpha \equiv \beta-2(1-k) m>0 \text { and } \alpha^{\prime}=\beta-(1-k) m>\alpha,
$$

for all $x$ in $D^{\prime}$, we have

$\|x-H(x)\| \geq\|x-T(x)\|-\|T(x)-H(x)\| \geq \beta-(1-k) m \equiv \alpha^{\prime}>0$.

Hence $\operatorname{deg}\left(I-H, D_{i}, 0\right)$ is well defined and equals $\operatorname{deg}\left(I-T, D_{i}, 0\right)$ for $i=1,2$. Let $\tilde{T}_{n}$ be the condensing mapping of $\bar{D}$ into $X$ defined by

$$
\tilde{T}_{n}(x)=T_{n}(x)+T\left(x^{*}\right)-T_{n}\left(x^{*}\right) \quad(x \in \bar{D}),
$$

where $x^{*}$ is any given point in $F(T)$. It follows from (1) and condition (a) that there exists an integer $N_{0} \geqq 1$ such that for each $n \geqq N_{0}, \widetilde{T}_{n}(x) \neq x$ for all $x$ in $D^{\prime}$. Indeed, (a) implies the existence of an integer $N_{0} \geqq 1$ such that $\alpha-2 \delta_{n} \geqq \alpha / 2$ for $n \geqq N_{0}$ and therefore for all $x$ in $D^{\prime}$ and $n \geqq N_{0}$, in view of (1), we have

$$
\begin{aligned}
\left\|x-\tilde{T}_{n}(x)\right\| & \geqq\|x-T(x)\|-\left\|T(x)-\tilde{T}_{n}(x)\right\| \\
& \geqq\|x-T(x)\|-\left\|T(x)-T_{n}(x)\right\|-\left\|T\left(x^{*}\right)-T_{n}\left(x^{*}\right)\right\| \\
& \geqq \beta-2 \delta_{n}>\alpha-2 \delta_{n}>\alpha / 2 .
\end{aligned}
$$

Hence $\operatorname{deg}\left(I-\tilde{T}_{n}, D_{i}, 0\right)$ is well defined for each $n \geqq N_{0}$ and $i=1,2$. Now, for each $n \geqq N_{0}$, consider the homotopy

$$
H_{n t}(x) \equiv H_{n}(x, t)=t \widetilde{T}_{n}(x)+(1-t) H(x), \quad x \in \bar{D}, t \in[0,1] .
$$

Since $T(\bar{D})$ is bounded and, by (a), $\left\{T_{n}(\bar{D})\right\}$ is uniformly bounded, it follows that $H_{n}(x, t)$ is continuous in $t \in[0,1]$, uniformly for $x$ in $\bar{D}$. Furthermore, for each fixed $n, H_{n}(\cdot, t)$ is a condensing mapping for each $t \in[0,1]$ because it is a convex combination of the condensing mappings 
$\widetilde{T}_{n}$ and $H$. Thus, by Lemma $1(\mathrm{~d})$, since $H_{n}((A \times[0,1])) \leqq \gamma(A)$ for each $A \subset \bar{D}$ and $n$, to show that $\operatorname{deg}\left(I-H_{n 0}, D_{i}, 0\right)=\operatorname{deg}\left(I-H, D_{i}, 0\right)=$ $\operatorname{deg}\left(I-H_{n 1}, D_{i}, 0\right)$ for each $n \geqq N_{0}$ and $i=1,2$, it suffices to show that $\left\|x-H_{n t}(x)\right\| \geqq \eta$ for $x \in D^{\prime}$ and $t \in[0,1]$ and some $\eta>0$. But, for each $n \geqq N_{0}$ and all $x$ in $D^{\prime}$ and $t$ in $[0,1]$, we have the relation

$$
\begin{aligned}
\left\|x-H_{n t}(x)\right\| \geqq & \|x-T(x)\|-\|T(x)-k T(x)\|-t\left\|\tilde{T}_{n}(x)-k T(x)\right\| \\
\geqq & \beta-(1-k) m \\
& -t\left\|T_{n}(x)-T(x)+T(x)-k T(x)+T\left(x^{*}\right)-T_{n}^{*}(x)\right\| \\
\geqq & \beta-(1-k) m-t(1-k) m-t 2 \delta_{n} \\
\geqq & \beta-2(1-k) m-2 \delta_{n}=\alpha-2 \delta_{n} \geqq \alpha / 2 \equiv \eta .
\end{aligned}
$$

Thus, by Lemma 1 (d), $\operatorname{deg}\left(I-H, D_{i}, 0\right)=\operatorname{deg}\left(I-H_{n 0}, D_{i}, 0\right)=$ $\operatorname{deg}\left(I-H_{n 1}, D_{i}, 0\right)=\operatorname{deg}\left(I-\tilde{T}_{n}, D_{i}, 0\right)$ for each $n \geqq N_{0}$ and $i=1,2$.

Now, let $x^{*}$ be a fixed point in $F_{1}\left(\subset D_{1}\right)$ and let $n \geqq N_{0}$ also be fixed. It follows from the definition of $\widetilde{T}_{n}$ in (2) that $\widetilde{T}_{n}\left(x^{*}\right)=T\left(x^{*}\right)=x^{*}$, i.e., $x^{*}$ satisfies the equation $x=T_{n}(x)+T\left(x^{*}\right)-T_{n}\left(x^{*}\right)$ and therefore, since $\left\|T\left(x^{*}\right)-T_{n}\left(x^{*}\right)\right\| \leqq \delta_{n}$, condition (b) implies that $x^{*}$ is the only fixed point of $\tilde{T}_{n}$. Hence $0 \notin\left(I-\tilde{T}_{n}\right)\left(\bar{D}_{2}\right)$, and consequently, by Lemma 1 (b) or its corollary for condensing mappings, $\operatorname{deg}\left(I-T_{n}, D_{2}, 0\right)=$ $\operatorname{deg}\left(I-H, D_{2}, 0\right)=0$, i.e., $\operatorname{deg}\left(I-T, D_{2}, 0\right)=0$. Similarly, taking $x^{*}$ in $F_{2}\left(\subset D_{2}\right)$ and using the same argument, one shows that

$$
\operatorname{deg}\left(I-T, D_{1}, 0\right)=0 .
$$

This contradiction shows that $F(T)$ is connected and thus a continuum. Q.E.D.

An immediate corollary of Theorem 1 is Theorem 5 in [2], stated here as Corollary 1.

COROLlaRY 1. If $T: \bar{D} \rightarrow X$ is a condensing mapping such that $\operatorname{deg}(I-T, D, 0) \neq 0$ and if there exists a sequence $\left\{T_{n}\right\}$ of condensing mappings of $\bar{D}$ into $X$ for which (a) and (b) of Theorem 1 hold, then $F(T) \subset D$ is a continuum.

Proof. Since every condensing map is 1 -set-contractive, to prove Corollary 1 it suffices to show that $T$ is demicompact if it is condensing. Now, let $\left\{x_{n}\right\}$ be a sequence in $\bar{D}$ such that $f_{n} \equiv x_{n}-T\left(x_{n}\right) \rightarrow f$ for some $f$ in $X$. Since a given set $S$ in $X$ is relatively compact if and only if $\gamma(S)=0$, it follows that $\gamma\left(\left\{f_{n}\right\}\right)=0$ and therefore, since $T$ is condensing, it follows that $\gamma\left(\left\{x_{n}\right\}\right)=0$, i.e., $\left\{x_{n}\right\}$ has a convergent subsequence. Q.E.D.

Another consequence of Theorem 1 is Theorem 4 in [2] which follows from Corollary 2 below for the case when $D=B(0, r)$, an open ball in $X$ with center at 0 and radius $r>0$. 
Corollary 2. Let $D$ be a bounded open subset of $X$ with $0 \in D$ and $T$ a demicompact 1-set-contraction of $\bar{D}$ into $X$ which satisfies condition $\left(\pi_{1}^{<}\right)$: If $T(x)=\alpha x$ holds for some $x$ in $\dot{D}$ then $\alpha<1$. Suppose there is $a$ sequence of condensing mappings $\left\{T_{n}\right\}$ of $\bar{D}$ into $X$ such that (a) and (b) of Theorem 1 hold then $F(T) \subset D$ is a continuum.

Proof. To show that Corollary 2 follows from Theorem 1, it suffices to show that $\operatorname{deg}(I-T, D, 0) \neq 0$. To establish the latter, by Lemma 1 (d), it suffices to show that the continuous mapping $F_{t}: \bar{D} \times[0,1] \rightarrow X$ defined by $F_{t}(x)=t T(x)$ for $x \in \bar{D}$ and $t \in[0,1]$ satisfies the conditions of Lemma 1 (d) for in that case we would have the looked for relation:

$$
\begin{aligned}
\operatorname{deg}(I-T, D, 0) & =\operatorname{deg}\left(I-F_{1}, D, 0\right)=\operatorname{deg}\left(I-F_{0}, D, 0\right) \\
& =\operatorname{deg}(I, D, 0)=1 \neq 0 .
\end{aligned}
$$

Since clearly $\gamma(F(A \times[0,1])) \leqq \gamma(A)$ for each $A \subset \bar{D}$, it suffices to show that there exists $\delta>0$ such that $\left\|x-F_{t}(x)\right\| \geqq \delta$ for $x$ in $\dot{D}$ and $t$ in $[0,1]$. If the latter inequality were not true for any $\delta>0$, then there would exist a sequence $\left\{x_{n}\right\} \subset \dot{D}$ and a sequence $\left\{t_{n}\right\} \subset[0,1]$ such that $x_{n}-$ $F_{t_{n}}\left(x_{n}\right) \rightarrow 0$ as $n \rightarrow \infty$. Assuming without loss of generality that $t_{n} \rightarrow t$ for some $t$ in $[0,1]$ we see that

$$
x_{n}-t T\left(x_{n}\right)=x_{n}-F_{t_{n}}\left(x_{n}\right)+\left(t_{n}-t_{n}\right) T(x) \rightarrow 0 \text { as } n \rightarrow \infty .
$$

Suppose first that $t=0$, then $x_{n} \rightarrow 0$ with $0 \in \dot{D}$, in contradiction to the assumption that $0 \in D$. Next, suppose that $t \in(0,1)$, then $t T$ is $t$-setcontraction with $t<1$ and therefore there exists a subsequence $\left\{x_{n_{j}}\right\}$ and $x$ in $\dot{D}$ such that $x_{n_{j}} \rightarrow x$ and $x-t T x=0$, in contradiction to the condition $\left(\pi_{1}^{<}\right)$satisfied by $T$ on $\dot{D}$. Finally, if $t=1$, then since $T$ is demicompact there exists a subsequence $\left\{x_{n_{j}}\right\}$ and $x$ in $\dot{D}$ such that $x_{n_{j}} \rightarrow x$ and $x-T(x)=0$, in contradiction to $\left(\pi_{1}^{<}\right)$. The above arguments show that Lemma $1(\mathrm{~d})$ is applicable. Q.E.D.

The author is indebted to P. M. Fitzpatrick for his useful observations during our study of the nature of fixed point sets.

\section{REFERENCES}

1. R. D. Nussbaum, The fixed point index for local condensing maps, Ann. Mat. Pura Appl. (to appear).

2. W. V. Petryshyn, Structure of the fixed point sets of $k$-set-contractions, Arch. Rational Mech. Anal. 40 (1971), 312-328.

3. M. Furi and A. Vignoli, A fixed point theorem in complete metric spaces, Boll. Un. Mat. Ital. (4) 4/5 (1969), 505-509.

Department of Mathematics, Rutgers University, New Brunswick, New JERSEY 08903 